\title{
Inter-symbol Interference Suppression Employing Sub-carrier Group Selection for OFDM-TDD Transmit Diversity
}

\author{
Fumiaki Maehara, Takeshi Ikenaga, Fumio Takahata*, and Satoshi Goto \\ Graduate School of Information, Production and Systems, Waseda University, Fukuoka, 808-0135 Japan \\ *School of Science and Engineering, Waseda University, Tokyo, 169-8555 Japan \\ Email: fumiaki@toki.waseda.jp
}

\begin{abstract}
This paper proposes an inter-symbol interference (ISI) suppression scheme using the sub-carrier group selection for orthogonal frequency division multiplexing (OFDM) time division duplex (TDD) transmit diversity. Although transmit diversity is quite effective in improving the transmission performance, the ISI due to the long multipath delays causes the error floor and leads to a loss of the space diversity benefit. To cope with this degradation, the periodic time domain waveform obtained by the even-numbered sub-carrier group is used for the extension of the guard interval. Moreover, the odd-numbered sub-carrier group is also applied to exploit the frequency diversity benefit, which is realized by carrying out the simple frequency conversion at the receiver. To keep the constant transmission rate even in the subcarrier group transmission, the high-level modulation scheme with power enhancement is applied. The computer simulation results show that the proposed scheme not only prevents the error floor due to the ISI but also achieves the space and frequency diversity benefit without the extension of the guard interval.
\end{abstract}

\section{INTRODUCTION}

In recent years, orthogonal frequency division multiplexing (OFDM) has become a promising technique for broadband wireless communication because of its applicability to high data rate transmission, bandwidth efficiency, and mitigation of the effects of the delay spread [1], [2]. To improve the bit error rate (BER) further, OFDM in combination with a transmit diversity technique is quite effective because additional cost at the receiver, as well as additional bandwidth is not required. Especially, time division duplex (TDD) transmit diversity [3] is attractive technique because a closed loop which performs better than a open loop can be realized without the feedback information (FBI).

On the other hand, when the multipath delays exceed the duration of the guard interval, its BER is deteriorated significantly due to the inter-symbol interference (ISI), which causes the error floor and leads to a loss of the space diversity benefit. To deal with this problem, the modified frame structure which extends a guard interval has been proposed [4], [5]. However, this approach not only decreases the transmission rate but also requires the change of the synchronization mechanism at the receiver. As for the simple ISI suppression without the transmission rate, the half symbol using even-numbered subcarriers is useful for the extension of the guard interval because its time waveform becomes periodic [6]. So far, we have proposed the half symbol transmission scheme using highlevel modulation [7], [8]. In [8], the odd-numbered sub-carrier is additionally utilized for the sub-carrier group selection, which creates the frequency diversity effect. If this scheme is applied to the OFDM-TDD transmit diversity, the space and frequency diversity effects can be exploited at the same time while eliminating the ISI perfectly.

This paper presents the ISI suppression scheme employing the half symbol transmission for OFDM-TDD transmit diversity. In the proposed scheme, the periodic time domain waveform generated by the even-numbered sub-carrier group is basically used for extending the guard interval [6]. In addition, the frequency conversion at the receiver makes the oddnumbered sub-carrier group applicable [8]. The main feature of the proposed scheme is to select not only the sub-carrier group but also the antenna according to the instantaneous channel condition, which exploits the space and frequency diversity benefit at the same time. Here, it should be noted that the transmission rate due to the use of the half symbol is compensated by applying the high-level modulation, which results in the constant transmission rate. We also present the computer simulation results to demonstrate the effectiveness of the proposed scheme under severe ISI conditions.

The following section describes the principle of the proposed ISI suppression scheme. Section 3 provides the simulation parameters and the BER performance of the proposed scheme. Finally, Section 4 summarizes this paper.

\section{Proposed Scheme}

\section{A. Half Symbol Transmission using Sub-carrier Group Selec- tion}

To eliminate the ISI, the cyclic prefix is intentionally inserted between successive OFDM symbols. However, the ISI occurs when the multipath delay is beyond the duration of the guard interval $T_{G}$. To cope with this problem, the half symbol transmission using the even-numbered sub-carriers is applied to the proposed scheme.

In OFDM transmission using a discrete Fourier transform (DFT) size of $N, N$ modulated sub-carriers with different frequencies are summed up by the inverse DFT (IDFT). Defining $T_{S}$ as the symbol duration of the IDFT, the sample 
duration $\Delta T$ is $T_{S} / N$ and all the sub-carriers are exactly spaced by $1 / T_{S}$ in the frequency band.

The OFDM symbol composed of only the even-numbered sub-carriers at $n \Delta T$ is given by

$$
s(n \Delta T)=\sum_{i=0}^{N^{\prime}-1} d(2 i) e^{j 2 \pi\left(\frac{i}{N^{\prime}}\right) n} .
$$

where $d(k)$ is the modulated signal and $N^{\prime}$ is defined as $N / 2$. From Eq. (1), it can be seen that an OFDM symbol composed of only the even-numbered sub-carriers is defined as the periodic signal with the period of $N^{\prime}(=N / 2)$. This fact implies that the first half of the OFDM signal can be considered as the cyclic prefix, while the second half of the OFDM signal can be used for demodulation. The half symbol transmission scheme utilizes this characteristic. In this scheme, the OFDM transmission using all sub-carriers is employed (full-symbol mode) under the ISI-free condition, where the maximum multipath delay $\tau_{\max }$ does not exceed the guard interval $\left(\tau_{\max }<T_{G}\right)$. In the ISI condition $\left(\tau_{\max }>T_{G}\right)$, the OFDM signal with the even-numbered sub-carriers is generated and only the second half of the OFDM symbol is demodulated (half-symbol mode). In the half symbol transmission, the number of samples is reduced to $N^{\prime}$, and therefore the number of bits per sub-carrier needs to be doubled to achieve the constant data rate. However, each sub-carrier power can be enhanced to twice as much as that of the full symbol mode from the point of view of the constant power transmission. Thus, if $M$-ary modulation is applied to the full symbol mode, the half symbol transmission uses $M^{2}$-ary modulation with the doubled sub-channel power.

In the half symbol transmission, the use of the oddnumbered sub-carrier group makes the sub-carrier group selection available, which creates the frequency diversity benefit [8]. In general, the channel conditions of the even- and oddnumbered sub-carrier groups are different under the frequency selective fading channels. Therefore, if the sub-carrier group with the lower average BER is chosen according to the channel condition, the frequency diversity benefit can be obtained at the expense of the additional frequency conversion at the receiver. Here, the simple frequency conversion changes the odd-numbered sub-carrier group into the even-numbered subcarrier group, which succeeds in giving the periodic characteristic to the odd-numbered sub-carrier group. Therefore, in the case of the odd-numbered sub-carrier transmission, the time domain signal is multiplied by $\exp \left(-j 2 \pi \frac{n}{N}\right)$ in order to give the frequency shift $\frac{n}{N}$, as expressed by

$$
r_{\text {even }}(n)=r_{\text {odd }}(n) \exp \left(-j 2 \pi \frac{n}{N}\right)
$$

where $r_{\text {even }}(n)$ and $r_{\text {odd }}(n)$ are the signals for the even- and odd-numbered sub-carrier groups, respectively.

In this way, the half symbol transmission using sub-carrier group selection creates the frequency diversity effect, as well as the perfect suppression of the ISI.

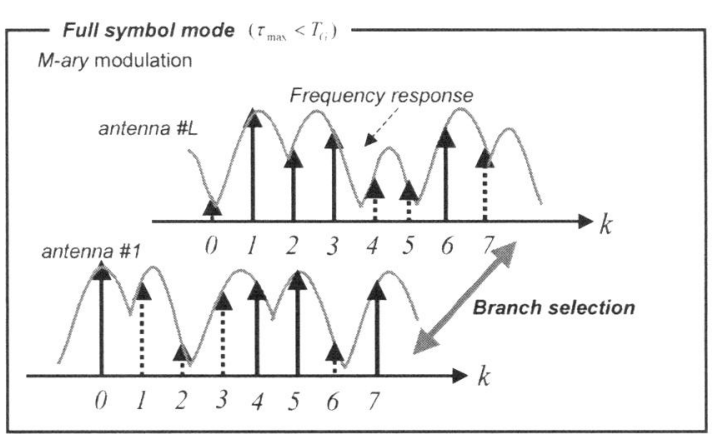

(a) Full symbol mode $\left(\tau_{\max }<T_{G}\right)$

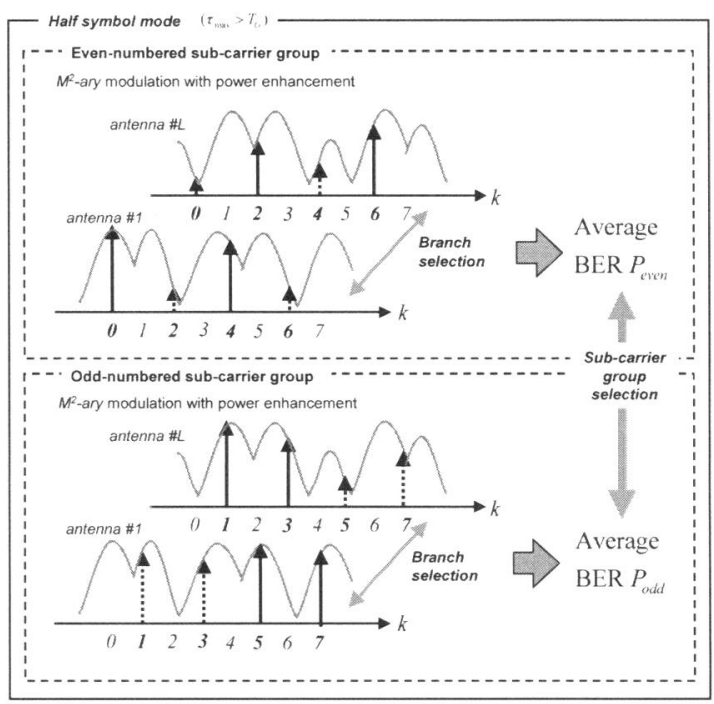

(b) Half symbol mode $\left(\tau_{\max }>T_{G}\right)$

Fig. 1. Concept of the proposed downlink sub-channel combining for $N=8$.

\section{B. Adaptive Transmit Diversity with Branch and Sub-carrier Group Selection}

Since TDD which makes use of the same carrier frequency between up- and down-links is applied as a duplex scheme, a transmit diversity technique can act as a closed loop which has more advantage than an open loop in terms of the BER performance. This is because the reversible channel characteristics can be obtained, and the base station (BS) can measure the channel condition required for the down-link transmission. In OFDM transmit diversity, a sub-channel combining is required for exploiting the space diversity benefit because a sub-channel experiences the flat fading even in the frequency selective fading channels.

Figure 1 shows the concept of the proposed downlink sub-channel combining for $N=8$ under a certain frequency selective fading channel. In the proposed scheme, the selection combining (SC) is adopted at the BS, and therefore the antenna with the largest instantaneous sub-channel power is selected based on the channel frequency response of the up- 
link transmission. In the ISI-free condition, the full symbol mode is performed, which is the same as the conventional OFDM transmit diversity with full sub-carriers. Therefore, at each sub-channel, the transmitting antenna with the largest power is selected. On the other hand, in the ISI condition, the proposed scheme makes use of the half symbol mode which utilizes the periodic time waveform to extend the guard interval. The half symbol mode handles either evennumbered sub-carrier group or odd-numbered one according to the channel condition, which creates the frequency diversity effect. Firstly, at each sub-channel, the transmitting branch is determined based on the SC. Then, the average BER of the selected branches for each sub-carrier group is calculated by using the estimated instantaneous CNR. In general, the BER of the $k$-th sub-carrier for the high-level modulation under AWGN conditions is given by [9]

$$
P_{e}(k)=a \operatorname{erfc}\left(\sqrt{b \gamma_{k}}\right),
$$

where $\gamma_{k}$ is the instantaneous CNR of the $k$-th sub-carrier at the selected branch, and both $a$ and $b$ are the coefficients given by the modulation scheme adopted as the high-level modulation. by

Thus, the average BER for each sub-carrier group is given

$$
\begin{gathered}
P_{\text {even }}=\frac{1}{N^{\prime}} \sum_{i=0}^{N^{\prime}-1} P_{e}(2 i) \\
P_{\text {odd }}=\frac{1}{N^{\prime}} \sum_{i=0}^{N^{\prime}-1} P_{e}(2 i+1) .
\end{gathered}
$$

The average BERs for both sub-carrier groups are compared to choose an appropriate sub-carrier group with the lower BER adaptively, which exploits the frequency diversity benefit, as well as the space diversity benefit. Therefore, the space and frequency diversity benefit can be achieved without the degradation due to the ISI, while the high-level modulation causes a certain BER degradation in general. Thus, the proposed scheme can be seen as a technique combining two kinds of diversity effects at the expense of the degradation due to the use of the high-level modulation. As a result, the proposed approach is advantageous when the combined diversity effect exceeds this degradation.

\section{System Configuration}

The uplink frame is based on the format, which has the two pilot symbols only in the head [10]. Here, the duration of the guard interval for the pilot symbols is twice as large as that for the following data symbols $(=N \Delta T / 4)$, where the ISI can be perfectly avoided at the BS. The channel estimates are used for not only the compensation of the up-link data symbols but also the determination of the transmission mode for the down-link transmission.

Figure 2 shows the configuration of the proposed scheme. At the BS, the guard interval is removed from the up-link received signal at each branch and each signal is converted

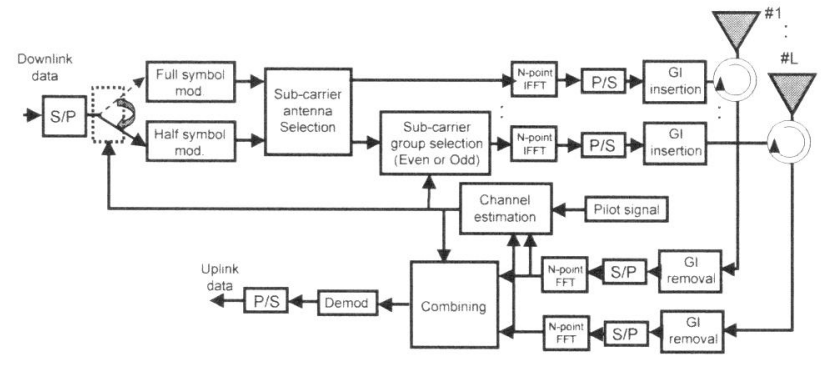

(a) Base station

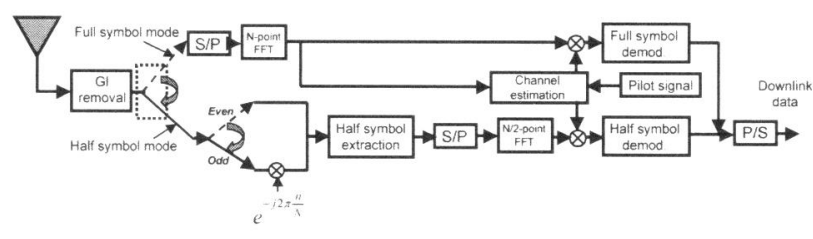

(b) Mobile station

Fig. 2. Overall configuration of proposed scheme.

into the $N$-sub-channel signals by $N$-point FFT processing. The channel frequency response at each antenna is estimated by using the pilot symbols. Since the multipath delays have to be estimated to judge whether the current channel condition can be seen as the ISI or not, the delay profile is required and then derived by the IFFT processing of the channel frequency response [7]. In the ISI condition, the half symbol mode is conducted and the proper sub-carrier group is determined by comparing the average BERs of both sub-carrier groups. As mentioned earlier, the derivation of the average BER requires measuring the instantaneous $\mathrm{CNR}$ of each sub-carrier. If the $m$-th branch is selected, the instantaneous CNR of $k$-th subchannel is given by

$$
\gamma_{k}=\frac{\left|H_{m}(k)\right|^{2}}{2 \sigma^{2}}
$$

where $\sigma^{2}$ and $H_{m}(k)$ are the noise variance and the channel frequency response of the $k$-th sub-carrier at the $m$-th branch, respectively. Here, both $\sigma^{2}$ and the difference in the transmitting power between the BS and the MS are assumed to be known at the BS.

In the ISI-free condition, the full symbol mode using all sub-carriers is performed. Once the transmission mode is fixed, the modulated signals are mapped to the respective subchannel and branch followed by the $N$-point IFFT processing. Finally, the guard interval is added to the IFFT output of each antenna. Here, it should be noted that the 2-bit information is needed at most to inform the MS of both the ISI condition and the appropriate sub-carrier group. However, considering the frame-based transmission, its impact on the transmission efficiency is quite small enough to be neglected.

At the MS, the received signal is switched according to the information of the transmission mode after removing the 
TABLE I

DELAY PROFILE OF EACH RMS DELAY SPREAD

\begin{tabular}{|c|c|c|c|c|c|c|c|}
\hline \multicolumn{2}{|c|}{ Tap number $[\Delta T]$} & 0.0 & 3.0 & 6.0 & 9.0 & 12.0 & 15.0 \\
\hline \hline \multirow{4}{*}{$\begin{array}{c}\text { Average } \\
\text { power } \\
{[\mathrm{dB}]}\end{array}$} & $\tau_{r m s}=\Delta T$ & 0.0 & -10.0 & -20.0 & -30.1 & -40.1 & -50.1 \\
\cline { 2 - 8 } & $\tau_{r m s}=2 \Delta T$ & 0.0 & -5.9 & -11.8 & -17.8 & -23.7 & -29.6 \\
\cline { 2 - 8 } & $\tau_{r m s}=3 \Delta T$ & 0.0 & -4.0 & -7.9 & -11.8 & -15.8 & -19.7 \\
\cline { 2 - 8 } & $\tau_{r m s}=4 \Delta T$ & 0.0 & -2.5 & -4.9 & -7.4 & -9.8 & -12.3 \\
\cline { 2 - 8 } & $\tau_{r m s}=5 \Delta T$ & 0.0 & -0.8 & -1.6 & -2.4 & -3.2 & -4.0 \\
\hline
\end{tabular}

guard interval. In the half symbol mode, the odd-numbered sub-carrier group requires the frequency conversion described by Eq. (2) so as to create the periodic signals. After recovering the even-number sub-carriers irrespective of the sub-carrier group, the second half of the symbol is extracted to avoid the ISI. Then, the extracted symbol is converted into the $N / 2$-sub-channel signals by $N / 2$-point FFT processing and is compensated by the $N / 2$-point channel frequency response obtained by the channel estimates for the full symbol mode. As for the full symbol mode, the compensation of the received signal is the same as the traditional OFDM demodulation using $N$-point FFT processing.

\section{iII. Performance Evaluation}

We compare the proposed scheme with the half symbol scheme using only the even-numbered sub-carrier group and the conventional full symbol scheme in terms of the BER performance.

\section{A. Simulation Parameters}

The number of branches $L$ is set to 2, 3 and 4, and the 2-bit transmission $(M=4)$ is assumed for our simulation, where QPSK and 16QAM are used for the full symbol mode and half symbol one, respectively. Hence, the coefficients $a$ and $b$ in Eq. (3) correspond to $3 / 8$ and $1 / 10$, respectively. The duration of the guard interval $T_{G}$ at data symbols is set to $8 \Delta T$ for $N=32$. The number of down-link pilot symbols is set to 2 and the time difference between up- and down-links $T_{D}$ is the parameter which represents the fading correlation between both links. The block fading, where the time variation does not occur during the frame, is assumed. The delay profile of each rms delay spread $\tau_{r m s}$ is shown in Table 1 , which assumes a 6-ray exponentially-decaying Rayleigh fading. The perfect frequency and symbol synchronization is assumed.

\section{B. BER Performance versus Average CNR $\Gamma$}

Figure 3 shows the comparison, in terms of the BER performance versus the average $\mathrm{CNR} \Gamma$, between the proposed scheme and other two schemes, where $L=2$ and the normalized maximum Doppler frequency $f_{D} T_{D}=2.4 \times 10^{-4}$. In Fig. 3 , the theoretical BERs of transmit diversity for QPSK and 16QAM under the flat fading channels are also shown for reference. When $\tau_{r m s}=\Delta T$, all schemes have almost the same BER because the ISI-free condition mostly forces the full symbol mode irrespective of the scheme. On the other hand, when $\tau_{r m s}=5 \Delta T$, the proposed scheme has better BER than other two schemes. In particular, it is interesting to see that, in a larger $\Gamma$, the BER of the proposed scheme is superior to that for QPSK in spite of the use of 16QAM. It indicates that the sub-carrier group selection creates the frequency diversity benefit, which combat the degradation due to the use of the high-level modulation.

\section{BER Performance versus Delay Spread $\tau_{r m s}$}

Figure 4 shows the comparison, in terms of the BER performance versus $\tau_{r m s}$, between the proposed scheme and other two schemes, where $L=2$ and $f_{D} T_{D}=2.4 \times 10^{-4}$. In Fig. 4, it can be found that, in the case that $\tau_{r m s}=5 \Delta T$ and $\Gamma=30 \mathrm{~dB}$, the proposed scheme has far better BER than other two schemes. This is because the increase in $\tau_{r m s}$ makes the channel conditions of both sub-carrier groups uncorrelated to each other, which enhances the frequency diversity benefit. Moreover, it can be observed that, thanks to the frequency diversity effect, the proposed scheme alleviates the drawback of the use of the high-level modulation as $\tau_{r m s}$ increases.

\section{Effect of Normalized Maximum Doppler Frequency $f_{D} T_{D}$ on $B E R$}

Here, from the practical point of view, it is important to evaluate the effect of the time difference between the up- and down-links on the BER. Figure 5 shows the BER performance versus the normalized maximum Doppler frequency $f_{D} T_{D}$, where $L=2, \tau_{r m s}=5 \Delta T$, and $\Gamma=30 \mathrm{~dB}$. From Fig. 5 , it is found that the BERs of all schemes get worse as $f_{D} T_{D}$ increases. This is because the increase of $f_{D} T_{D}$ makes the fading correlation between both links lower, which reduces the space diversity benefit. In addition, the appropriate subcarrier group selection does not work well under the low fading correlation. Therefore, $T_{D}$ needs to be short enough to keep the high fading correlation between both links.

\section{E. Effect of the Number of Branches $L$ on BER}

Figure 6 shows the BER performance versus the the number of branches $L$, where $\Gamma=25 \mathrm{~dB}, \tau_{r m s}=5 \Delta T$, and $f_{D} T_{D}=$ $2.4 \times 10^{-4}$. From Fig. 6, it can be seen that the BERs of all schemes are improved with the increase in $L$ because the space diversity benefit is enhanced. In particular, since the proposed scheme provides the best performance irrespective of $L$, it turns out that the sub-carrier group selection contributes to the improvement in the BER. From these results, the effectiveness of the proposed scheme can be confirmed with respect to the number of branches.

\section{ConClusion}

We have proposed the ISI suppression scheme using the half symbol transmission for OFDM-TDD transmit diversity. In the proposed scheme, not only the sub-carrier group selection but also the antenna selection are employed to create the space and frequency diversity benefit. Numerical results given by computer simulations showed that the proposed scheme can exploit these two kinds of diversity benefits at the same time, while 


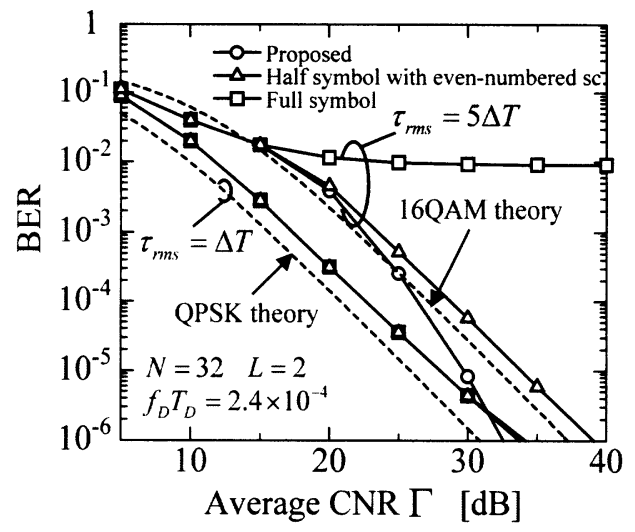

Fig. 3. Comparison, in terms of BER performance versus average CNR $\Gamma$, between proposed scheme and other schemes.

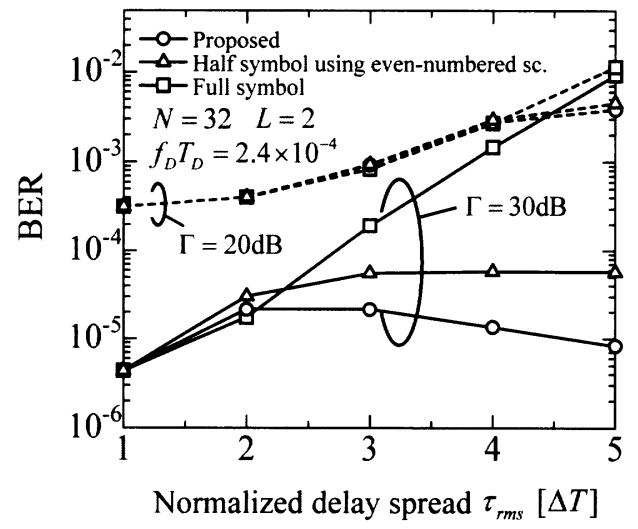

Fig. 4. Comparison, in terms of BER performance versus delay spread $\tau_{r m s}$, between proposed scheme and other schemes.

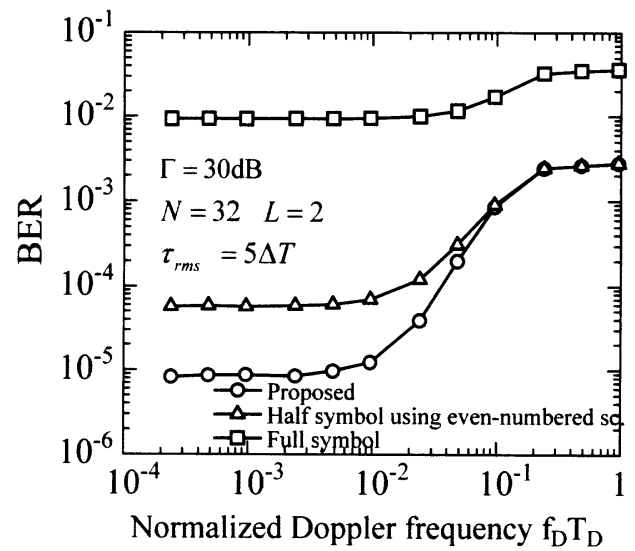

Fig. 5. Comparison, in terms of BER performance versus normalized maximum Doppler frequency $f_{D} T_{D}$, between proposed scheme and other schemes.

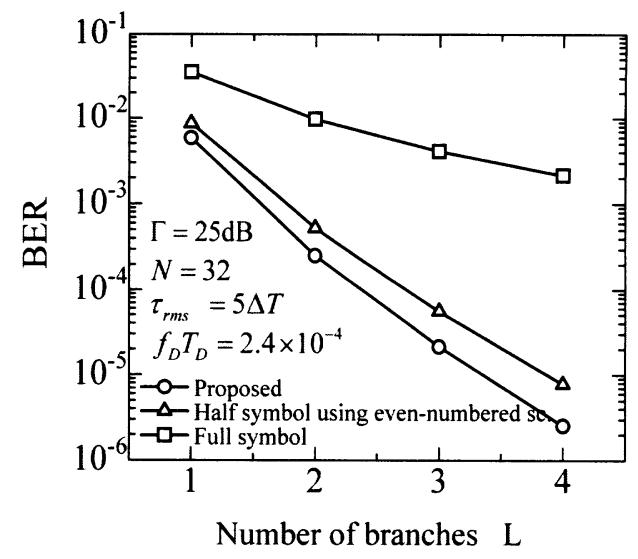

Fig. 6. Comparison, in terms of BER performance versus the number of branches $L$, between proposed scheme and other schemes.

suppressing the ISI and provide better BER performance than the half symbol transmission using only the even-numbered sub-carrier group and the traditional full symbol transmission under the severe ISI channels. The proposed scheme can be seen as a powerful technique to suppress the ISI completely while leaving the space diversity effect.

\section{ACKNOWLEDGMENT}

This work was supported by the Kitakyushu Human Techno Cluster projects funded by the Ministry of Education, Culture, Sports, Science and Technology of Japan.

\section{REFERENCES}

[1] V. Nee and R. Prasad, "OFDM for wireless multimedia communications," Artech House, 2000.

[2] S. Hara and R. Prasad, "Multicarrier techniques for 4G mobile communications," Artech House Publishers, 2003.

[3] Y. Matsumoto, N. Mochizuki, and M. Umehira, "OFDM subchannel space-combining transmission diversity for broadband wireless communication systems," IEEE ICUPC '98, pp 137-141, Oct. 1998.

[4] ETSI, "Digital broadcasting system for television, sound and data services; channel coding and modulation for digital terrestrial television," ETS 300744.

[5] Y. Asai, Y. Suzuki, and M. Umehira, "Adaptive coded OFDM system employing guard interval length control," Proc. of WPMC 2001, pp. 10711076, Sept. 2001.

[6] W. Matsumoto and H. Imai, "Study on half-symbol scheme of OFDM modulation and MC-CDMA modulation," IEICE Trans. Commun., vol. J85-B, no.6, pp. 910-921, June. 2002.

[7] F. Maehara, F. Sasamori, and F. Takahata, "Inter-symbol interference suppression scheme using even-numbered sub-carriers for fixed-rate OFDM systems," IEICE Trans. Commun., Vol.E87-B, No.4, pp. 866-872, April 2004.

[8] F. Maehara, H.-P. Kuchenbecker, "Inter-symbol interference suppression scheme employing sub-carrier group selection for fixed-rate OFDM systems," Proc. 9th International OFDM-Workshop, Sept. 2004.

[9] S. Sampei, "Applications of digital wireless technologies to global wireless communications," Prentice Hall PTR, 1997.

[10] IEEE P802.1 la, "High speed physical layer (PHY) in 5GHz band," 1999 